\title{
INFLUENCIA DEL RIESGO PAÍS EN EL RENDIMIENTO DE LOS BONOS SOBERANOS DE PERÚ
}

\author{
INFLUENCE OF COUNTRY RISK ON THE YIELD OF PERU'S SOVEREIGN BONDS
}

Winston Adrián Castañeda Vargas 1

ORCID: https://orcid.org/0000-0003-4257-1760 wcastaneda@esan.edu.pe

Walther Arturo Bueno Mariaca ${ }^{1}$

ORCID: https://orcid.org/0000-0002-7649-4591 walbueno@virtual.upt.pe

Gerardo Renato Arias Vascones ${ }^{1}$

ORCID: https://orcid.org/0000-0002-0123-1834 gerariasv@virtual.upt.pe

\section{RESUMEN}

La investigación desarrollada tiene como objetivo analizar el grado de influencia entre el riesgo país medido a través de EMBIG y el rendimiento de los bonos soberanos emitidos por el sector público de Perú. El estudio es de tipo longitudinal, para lo cual se toma información del Banco Central de Reserva del Perú y para comprobar los resultados se utilizó un modelo de regresión múltiple, con la finalidad de establecer el grado de causalidad entre ambas variables. Se concluyó que tanto la variable dependiente tratada como endógena y rezagada un periodo, así como el riesgo país, influyen significativamente en el rendimiento de los bonos soberanos emitidos por el Perú.

Palabras clave: Riesgo país y bonos soberanos

\section{ABSTRACT}

The research carried out aims to analyze the degree of influence between country risk measured through EMBIG and the yield of sovereign bonds

\footnotetext{
${ }^{1}$ Universidad Privada de Tacna. Facultad de Ciencias Empresariales
} 
issued by the public sector of Peru. The study is longitudinal, for which information is taken from the Central Reserve Bank of Peru and a multiple regression model was used to verify the results, in order to establish the degree of causality between both variables. It was concluded that both the dependent variable treated as endogenous and lagged one period, as well as the country risk, significantly influence the yield of sovereign bonds issued by Peru.

Keywords: Country risk and sovereign bonds.

\section{INTRODUCCIÓN}

Los países a menudo necesitan financiarse para poder ejecutar sus proyectos o implementar sus políticas públicas. Una de las formas de financiarse es a través de los organismos financieros internacionales como: Fondo Monetario Internacional (en adelante FMI), Banco Mundial (en adelante BM), entre otros. Sin embargo, los costos de financiamiento son un factor clave al momento de elegir un acreedor. Es por ello que, surge como alternativa de financiamiento la emisión de bonos soberanos, los cuales representan una forma de obtener financiamiento a medida y con menores costos.

De acuerdo con Levinson (2008), etimológicamente la palabra bono significa contrato y es utilizado por emisores (agentes deficitarios de dinero) para financiar proyectos, reestructurar deuda o diversificar pasivos (Moreno, 2014). Según Levinson (2008) citado por Moreno (2014), fue el Banco de Venecia quien emitió el primer bono del que se tenga registro y fue durante el siglo XII para costear la guerra contra Constantinopla. Los bonos son instrumentos de renta fija y representan deuda que debe ser pagada periódicamente o al final mediante cupones más el importe del nominal o facial al término del periodo establecido (Rodríguez, 2010). Dichos bonos pueden ser emitidos por empresas, países u organismos públicos que necesitan recursos para ejecutar sus proyectos o planes. (Pilbeam, 2018). Una ventaja de los bonos es que generalmente no necesitan garantías por parte del emisor como sí lo necesitan los créditos bancarios. (Horna, 2020)

Los bonos son valores de contenido crediticio, nominativos, representados mediante anotación en cuenta y libremente negociables. Son emitidos por la República del Perú, representada por el Ministerio de Economía y Finanzas (en adelante MEF) a través de la Dirección General de Endeudamiento y Tesoro Público. Los bonos emitidos localmente son denominados Bonos Soberanos (MEF, 2021). En el año 2003, el MEF estableció un programa de creadores de mercado (PCM), para desarrollar un mercado de deuda pública en moneda nacional, fomentando así el desarrollo de un mercado de capitales local. El PCM comprendía: i) la emisión de bonos soberanos en moneda nacional (BTP) a una tasa fija y a una tasa ajustada por inflación (poder adquisitivo constante, VAC); y ii) la negociación activa de la BTP en el mercado secundario que ha permitido la formación de la curva de rendimiento en moneda nacional, aumentando así la participación de la deuda pública en moneda nacional (Rossini, Montoro, \& Luna, 2020)

El 23 de noviembre de 2020, el Perú recurrió al financiamiento vía bonos para atender los gastos asociados a las medidas de lucha contra la COVID - 19. Asimismo, para seguir estimulando la reactivación económica y para financiar los gastos considerados en el presupuesto público 2020. La Oficina de Comunicaciones del Ministerio de Economía y 
Finanzas informó lo siguiente: "operación de endeudamiento a través de la emisión de tres nuevas referencias en dólares con vencimientos en los años 2032, 2060 y 2121, por un total de US\$ 4000 millones, obteniendo tasas cupones de 1,862\%, 2,78\% y 3,23\%, respectivamente" (Ministerio de Economía y Finanzas, 2020). La emisión de bonos a 100 años fue un hito histórico en la vida económica del país, dado que la confianza generada a nivel internacional, la estabilidad fiscal, el crecimiento del PBI, entre otros; han permitido captar el interés en los bonos emitidos al 2121 con tasas de interés competitivas y razonables.

Los bonos pueden clasificarse de acuerdo con el ente emisor, es decir, pueden ser: gobiernos nacionales, gobiernos a nivel inferior, empresas e instrumentos de titulización (bonos con garantía en activos) (Levinson, 2008). A nivel mundial, los bonos que tienen mayor confianza y mínimo riesgo son los bonos del tesoro de los Estados Unidos (Treasuries Bonds). (Moreno, 2014). De igual manera, Levinson (2008) citado por Moreno (2014) expone que los bonos también pueden clasificarse como: ordinarios (paga cupones periódicamente), redimibles (emisor puede amortizar de forma adelantada los bonos), no refinanciables (condiciones iniciales de emisión no cambian hasta el vencimiento), con opción de reventa (el tenedor puede revender los bonos al emisor), obligaciones perpetuas (bonos de plazo indefinido), cupón cero (los cupones se pagan al final junto con el nominal), convertibles (pueden ser intercambiados por otro título valor del emisor), interés variable (varía de acuerdo a las condiciones del mercado). Los bonos reciben una calificación de acuerdo con su nivel de riesgo, es decir, una clasificadora de riesgo evalúa las condiciones económicas y financieras del emisor para medir su capacidad de cumplir con los compromisos monetarios de los bonos (Moreno, 2014). Tal es así que existen diversos clasificadores de riesgo como: Feller Rate, Humphreys, Moody's, S \& P Global Ratings, Fitch Ratings, Dagong Global Credit Rating, entre otras. Según la evaluación del emisor, los bonos podrían tener las siguientes calificaciones:

Figura 1

Calificación de riesgo de los bonos

\begin{tabular}{|c|c|}
\hline c & DEFINICIÓN \\
\hline AAA & El emisor presenta una capacidad extremadamente fuerte para cumplir con sus compromisos financieros. \\
\hline A & $\begin{array}{l}\text { El emisor presenta una capacidad fuerte para cumplir con sus compromisos financieros, pero es un poco más susceptible } \\
\text { a condiciones y situaciones económicas adversas que los emisores calificados en categorías más altas }\end{array}$ \\
\hline $\mathrm{BBB}$ & $\begin{array}{l}\text { El emisor presenta una capacidad adecuada para cumplir con sus compromisos financieros. Sin embargo, condiciones } \\
\text { económicas adversas o cambios coyunturales probablemente conducirán al debilitamiento de su capacidad para cumplir } \\
\text { con sus compromisos financieros }\end{array}$ \\
\hline BB & $\begin{array}{l}\text { El emisor es menos vulnerable en el corto plazo, que otros emisores con calificaciones más bajas. Sin embargo, este } \\
\text { emisor enfrenta constante incertidumbre y exposiciones ante situaciones económicas y financieras adversas del negocio, } \\
\text { lo que puede hacer que su capacidad sea inadecuada para cumplir con sus compromisos financieros }\end{array}$ \\
\hline B & $\begin{array}{l}\text { El emisor es más vulnerable en el corto plazo que otros emisores con calificaciones BB, pero actualmente presenta } \\
\text { capacidad para cumplir con sus compromisos financieros. Condiciones adversas del negocio, financieras o económicas } \\
\text { probablemente perjudicarían la capacidad o voluntad del emisor para cumplir con sus compromisos financieros }\end{array}$ \\
\hline $\mathrm{R}$ & $\begin{array}{l}\text { El emisor está bajo supervisión regulatoria, debido a sus condiciones financieras. Durante la vigencia de ese proceso, los } \\
\text { reguladores podrían tener la facultad de favorecer a una clase de obligaciones por sobre otras o cumplir con el pago de } \\
\text { una obligación y no de otras }\end{array}$ \\
\hline SD y D & $\begin{array}{l}\text { El emisor no ha cumplido con el pago de una o más de sus obligaciones financieras (calificados o no). La calificación D } \\
\text { también se emplea tras la solicitud de bancarrota o en caso de una acción similar si los pagos de una obligación } \\
\text { financiera están en riesgo de no ser cubiertos. Se asigna una calificación D cuando Standar\&Poor`s considera que un } \\
\text { incumplimiento de pago será general y que el deudor no pagará puntualmente ninguna o casi ninguna de sus } \\
\text { obligaciones. Se atribuye una calificación SD cuando Standard \&Poor`s considera que se trata de un incumplimiento en } \\
\text { relación con una emisión específica o clase de obligaciones, pero que el emisor continuará honrando otras emisiones o } \\
\text { clases de obligaciones dentro de los plazos establecidos. }\end{array}$ \\
\hline NR & Al emisor no se le ha asignado calificación \\
\hline
\end{tabular}

Fuente: Moreno (Moreno, 2014, pág. 74) 
Los bonos soberanos que son emitidos por países requieren de una evaluación global de la economía del país emisor toda vez que el riesgo asociado al bono depende de la estabilidad y buena salud de las finanzas públicas. Una de las variables que afectan la calificación del bono es el riesgo país (Stowell, 2018). Para Santilli (2021) el riego país es: "un indicador que puede afectar el crecimiento y la estabilidad de una economía", lo cual se condice con lo expuesto por Gnecco (2008) quien define al riesgo país como el riesgo asociado al incumplimiento de deudas de un país subdesarrollado. Limas y Franco (2018) manifiestan que el riesgo país es un indicador que mide el riesgo que sufren las inversiones extranjeras ante el incumplimiento de acuerdos preestablecidos en un país emergente.

Rodríguez, Ayala e Iturralde (2003) manifestaron que las inversiones internacionales son claves para los países emergentes, por tal motivo, se debe tener especial cuidado del manejo económico ya que es fundamental para generar confianza a los mercados internacionales, los cuales prestan especial atención al riesgo país. Existen dos problemas principales cuando el riesgo país de una nación se incrementa, los cuales son: primero, anticipo de problemas económicos y posible impago de deuda externa; segundo, a mayor nivel de riesgo país mayor será el costo de financiación (Rodríguez, Ayala, \& Iturralde, 2003). En ese sentido, el riesgo país es un indicador de gran importancia dado que representa la calidad financiera y económica de un país en vías de desarrollo.

Al 25 de agosto de 2021, el banco de inversión JP Morgan estableció el riesgo país de Perú en 1.42 puntos porcentuales, el cual es uno de los más bajos de toda la región junto con Chile (128 pbs), Uruguay (158 pbs) y Colombia (270 pbs). El riesgo país más alto de Sudamérica lo tiene Venezuela (27800 pbs) seguido de Argentina (1586 pbs) y Ecuador (776 pbs). Es claro que la situación económica de Venezuela es crítica, razón por la cual los inversionistas extranjeros no llevan sus capitales hacia dicho país ya que no existe ninguna confianza en su economía ni en el régimen político actual. Argentina viene pasando por una crisis, aunque gracias al financiamiento del FMl viene recuperándose lentamente. Es necesario indicar que la pandemia ha golpeado a todos los países, a unos más que a otros, pero con los procesos de vacunación en marcha en todo el mundo, lentamente las economías vienen recuperándose, lo cual es positivo para los mercados internacionales que desean invertir en América Latina.

De lo expuesto anteriormente, es claro que un nivel bajo de riesgo país le permitirá a cualquier país emergente tener una exitosa emisión de bonos soberanos. Un ejemplo es el Perú con un bono emitido a cien años, lo cual indica la gran confianza que tienen las inversiones extranjeras en la solidez y estabilidad de la economía peruana. Gomero, Masuda y Barrera (2014) mencionaron que la demanda de los bonos va depender fundamentalmente del nivel de riesgo (riesgo país) asociado al emisor (país emergente), el cual de ser alto afectaría directamente a la tasa de interés (mayor riesgo mayor tasa), lo cual superaría las expectativas iniciales del emisor, es decir, el financiamiento sería más costoso al existir un mayor riesgo y menor confianza.

\section{MATERIALES Y MÉTODOS}

El presente estudio pretende determinar si el riesgo país influye en el rendimiento de los bonos soberanos peruanos. La investigación es pura o básica y de corte longitudinal ya que se extrajeron datos de más de un periodo. El enfoque es cuantitativo, el nivel es explicativo porque se trata de explicar la influencia de la variable independiente riesgo país sobre la variable dependiente rendimiento de los bonos peruanos. El diseño es no 
experimental toda vez que no se manipularon las variables en estudio. Las observaciones de las variables riesgo país y rendimiento de los bonos tienen periodicidad mensual y comprenden desde enero del 2014 hasta diciembre de 2020, siendo en total 84 observaciones, las cuales fueron extraídas de la base de datos del Banco Central de Reserva del Perú (BCRP). En ese sentido se tiene una población de 84 datos para ambas variables, los cuales serán procesados mediante el software Eviews 10.

\section{HECHOS ESTILIZADOS}

En la Figura 2, se muestra la participación de los bonos soberanos como porcentaje del producto bruto interno (PBI), en ese sentido se puede apreciar que este activo financiero emitido por el MEF tiene un comportamiento creciente, esto debido a los fundamentos macroeconómicos del Perú, así como, la clasificación de grado de inversión. De otro lado, al primer trimestre del 2021 la participación a disminuido al 17,11\%. En la Figura 3, se aprecia la participación de los inversionistas residentes y no residentes, respecto al año 2020 la participación de estos últimos fue del orden del 51.4\%, sin embargo, es necesario precisar que al primer trimestre del presente año dicha participación se redujo al $49 \%$, debido a la incertidumbre política que experimenta el país y a la tendencia alcista de las tasas de interés del banco central de Estados Unidos.

Figura 2

Bonos soberanos como proporción delPBI

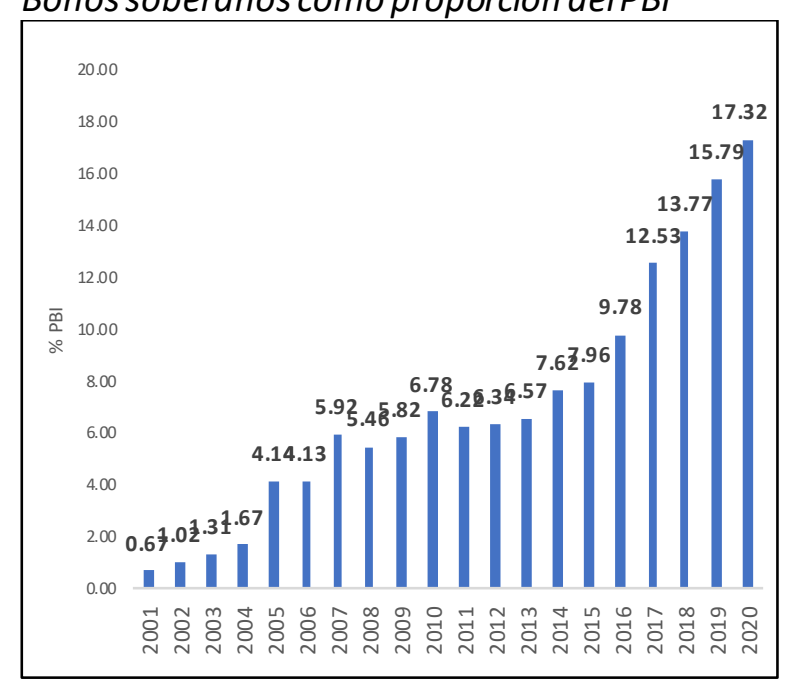

Figura 3

Saldo de los bonos soberanos por participantes

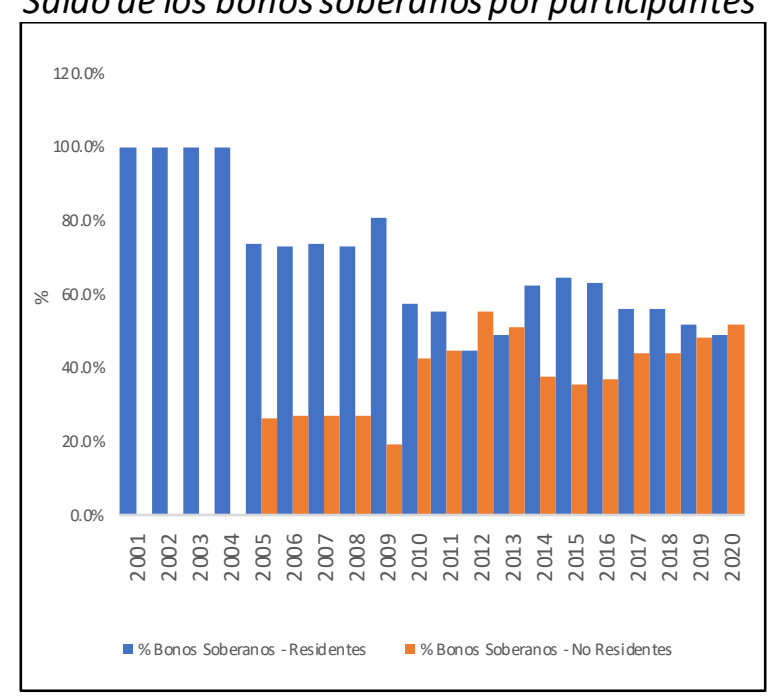

La Figura 4 muestra los saldos de los bonos soberanos del Perú al año 2020, donde se aprecia que asciende a 126,642 millones de soles, así mismo, al primer trimestre del presente año se incrementó la demanda en $1,79 \%$ respecto al último año. De otro lado, el activo financiero muestra un comportamiento exponencial debido a la confianza de los inversionistas, tanto residentes como no residentes. En la Figura 5, se observa la curva la curva de rendimiento de los bonos soberanos del Perú a diferentes plazos, que van desde dos años hasta 30 años. Se puede concluir en general que el rendimiento de los bonos ha sido creciente, por ejemplo, el bono a 20 años tiene una rentabilidad de 7,38\% actualmente. 
Figura 4

Saldo de Bonos soberanos (millones S/.)

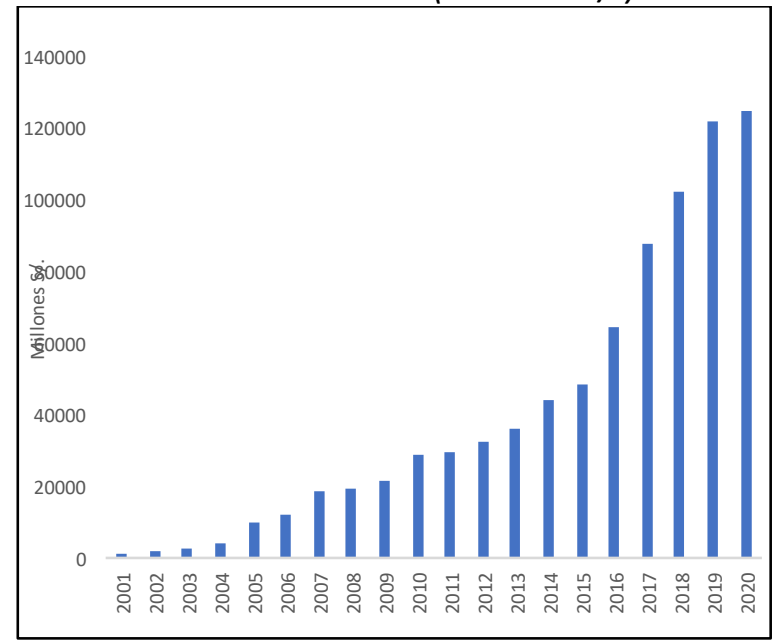

Figura 5

Curva de rendimiento del bono soberano

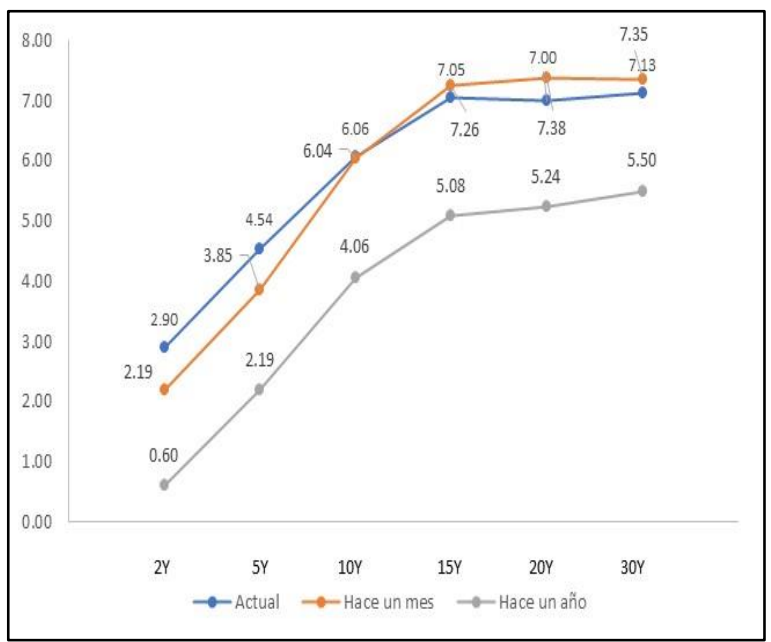

Del análisis comparativo de las curvas de rendimiento de Perú, México, Colombia, Brasil y Chile; se puede indicar que: hoy, hace un mes y hace un año; Perú tiene una tasa de rendimiento del bono a 10 años superior sólo a Chile del 6.10\%, 6.04\% y 4.06\% respectivamente, lo cual se aprecia en la Figura 6, mientras que en la Figura 7, se compara el rendimiento de los bonos soberanos a 20 años entre Perú y México. Al respecto, Perú se encuentra por debajo de México. Además, solo Perú y México son los únicos que han emitido bonos a 20 años.

\section{Figura 6}

Curva de rendimiento de los Bonos soberanos a 10 Años de América Latina

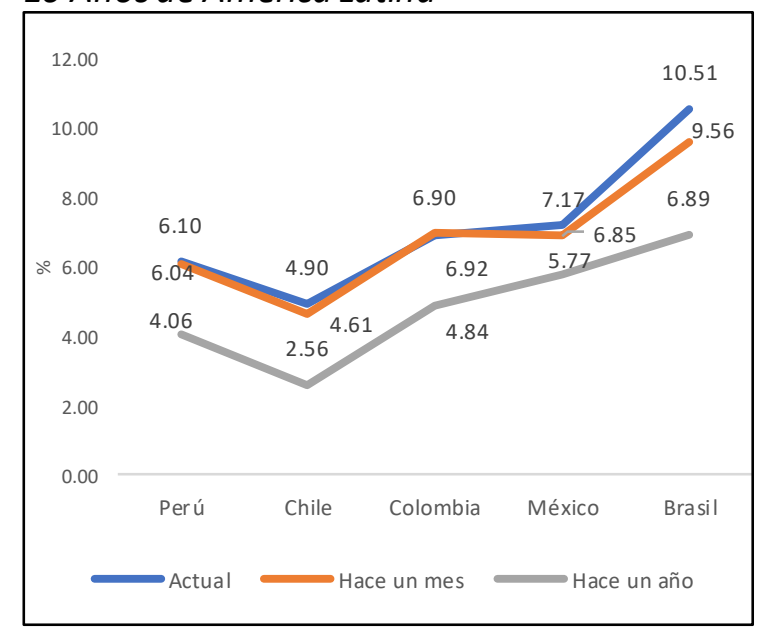

\section{Figura 7}

Curva de rendimiento del bono soberano a 20 años de Perú y México

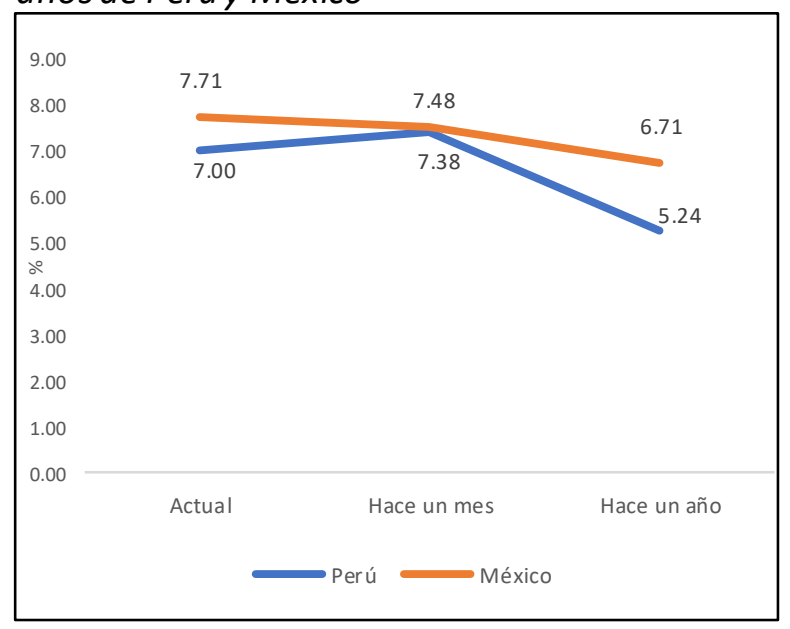

En la Figura 8, se aprecia el riesgo país de los países emergentes de América Latina, al mes de julio del presente año, se puede apreciar que el Perú juntamente con Chile tienen el menor riesgo país, es decir, el 143.7 y 170 puntos básicos. Esto muy relevante, dado que a menor riesgo país favorece la atracción de inversiones, así como, genera una mayor confianza 
que contribuye a una mayor demanda de activos financieros, como los bonos soberanos, dado el grado de confianza y grado de inversión del país.

Figura 8

Riesgo países de los países emergentes

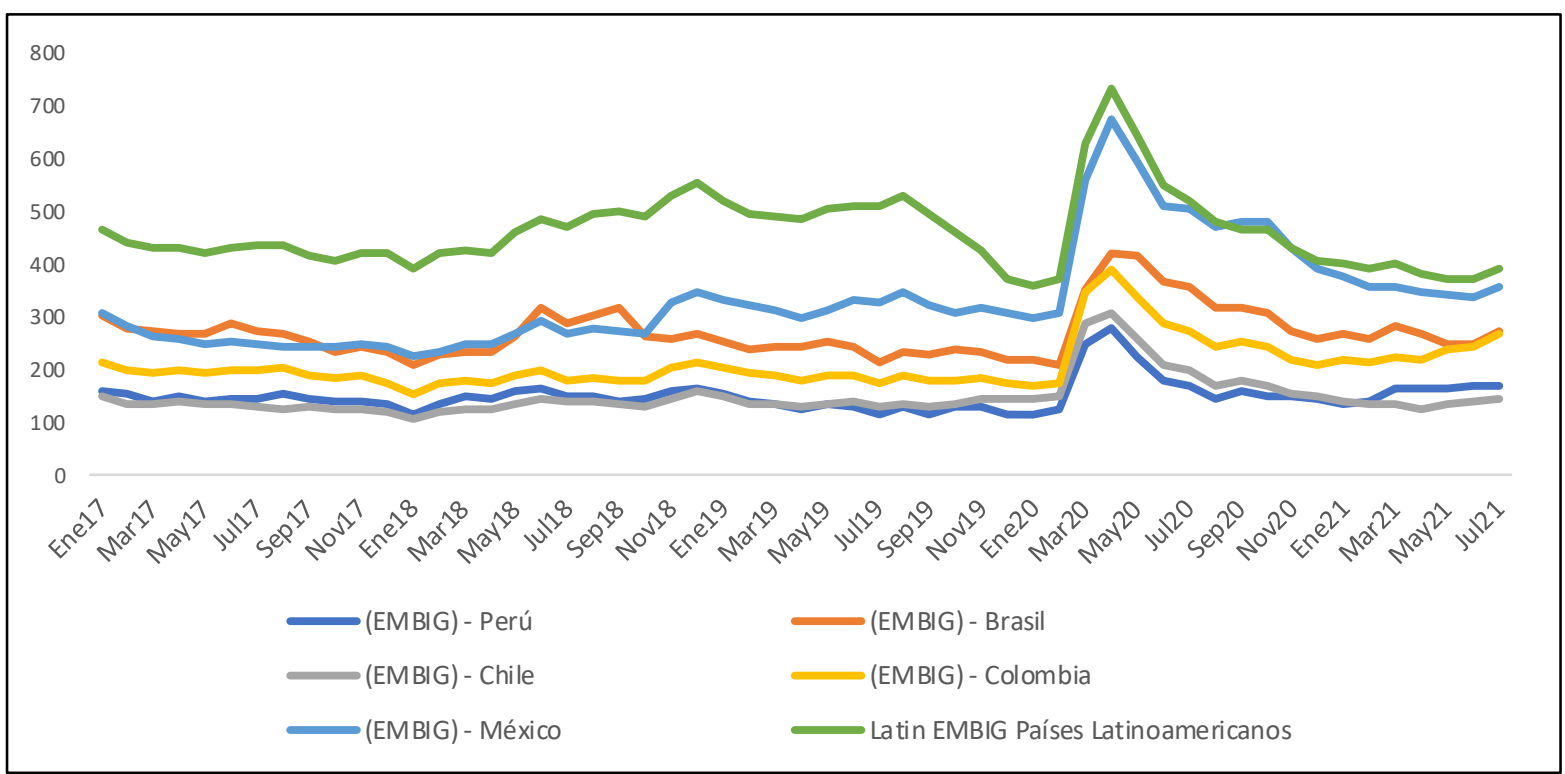

\section{RESULTADOS}

Con el propósito de demostrar los efectos que tiene el riesgo país en el rendimiento de los bonos soberanos emitidos por el Perú, se ha tomado en consideración el rendimiento de bonos emitidos a 20 años, durante el periodo de análisis del 01 de enero de 2014 al 21 de mayo de 2021, la data es longitudinal y mensual.

Tabla 1

\section{Modelo de regresión múltiple}

Dependent Variable: BONOS_SOBERANOS_20_ANOS

Method: Least Squares

Date: 09/01/21 Time: 19:35

Sample (adjusted): 2014M02 2021M05

Included observations: 88 after adjustments

\begin{tabular}{lclll}
\hline \hline \multicolumn{1}{c}{ Variable } & Coefficient & Std. Error & t-Statistic & Prob. \\
\hline \hline BONOS_SOBERANOS_20_ANOS(-1) & 0.893367 & 0.046243 & 19.31880 & 0.0000 \\
\multicolumn{1}{c}{ EMBIG(-1) } & -4.811681 & 1.468730 & -3.276082 & 0.0015 \\
& 497.7992 & 228.2370 & 2.181063 & 0.0320 \\
\hline \hline R-squared & 0.882361 & Mean dependent var & 6134.693 \\
Adjusted R-squared & 0.878159 & S.D. dependent var & 757.9857 \\
S.E. of regression & 264.5802 & Akaike info criterion & 14.03856 \\
Sum squared resid & 5880227. & Schwarz criterion & 14.15116 \\
Log likelihood & -613.6964 & Hannan-Quinn criter. & 14.08392 \\
F-statistic & 210.0155 & Durbin-Watson stat & 1.971922 \\
Prob(F-statistic) & 0.000000 & & \\
\hline \hline
\end{tabular}

Figura 9

Curva de rendimiento bono soberano a 20 años

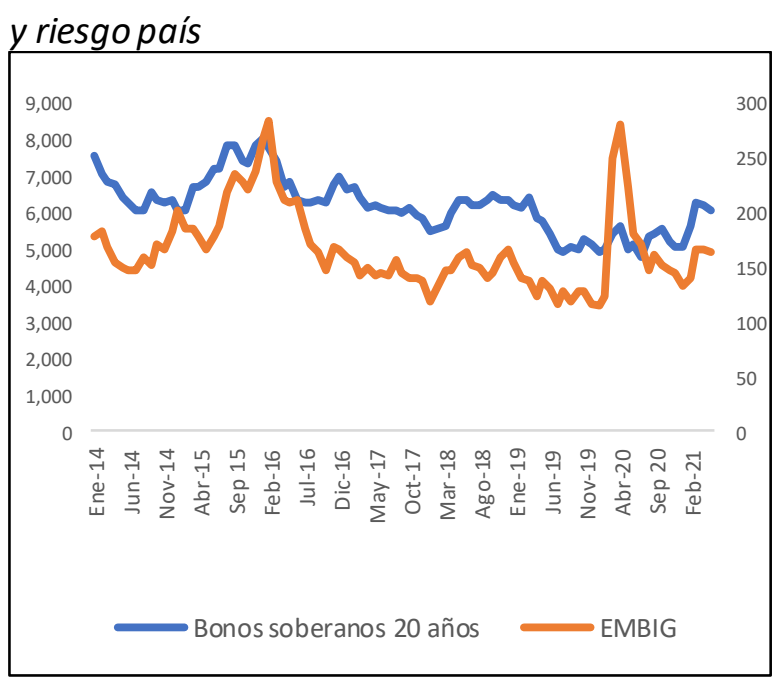


De la Figura 8, podemos concluir que el riesgo país es estadísticamente significativo, es decir, explica la variable bonos soberanos. Asimismo, esta relación es inversa, es decir, a medida que se incrementa el riesgo país el rendimiento del activo financiero disminuye, considerando que tiene un retardo de un periodo. De otro lado, la variable dependiente ha sido incorporada como variable independiente con un rezago, siendo esta también significativa, lo cual significa que el comportamiento de las variables en el pasado tiene un efecto en la variable rendimiento de los bonos de 20 años.

De otro lado, la Figura 9 muestra el grado de asociación entre ambas variables de estudio, cuya correlación de Pearson es de 59,2\%, es decir, existe una relación fuerte.

Figura 10

Serie de rendimiento de Bonos soberanos a 20 años en primeras diferencias

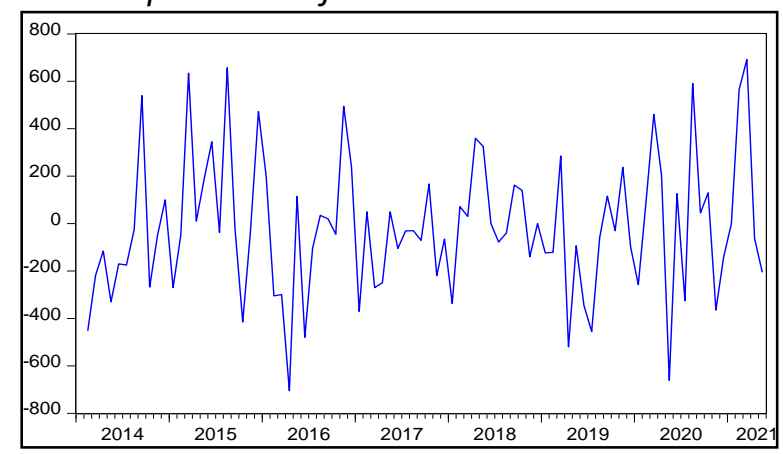

Figura 11

Series de riesgo país en primeras diferencias

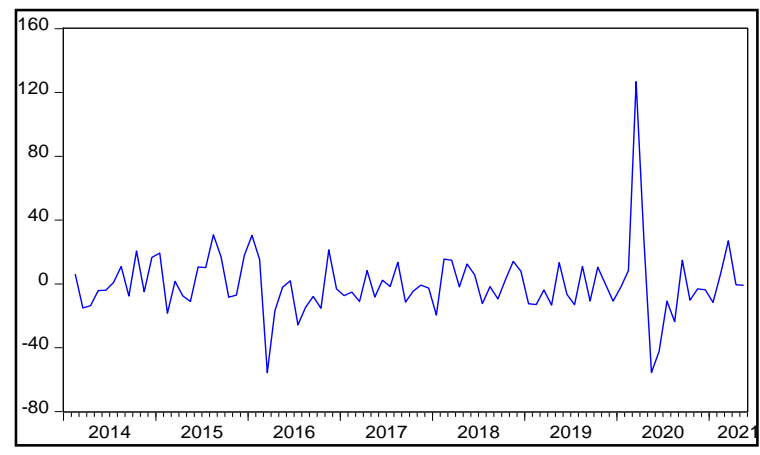

Dado que las series deben ser estacionarias se procedió a diferenciarlas en primeras diferencias, obteniéndose el resultado deseado, tal como se muestra en las Figuras 10 y 11. Para tomar esta decisión se efectuó el test aumentado de Dickey-Fuller.

Tabla 2

Test de autocorrelación de Breusch - Godfrey Breusch-Godfrey Serial Correlation LM Test:

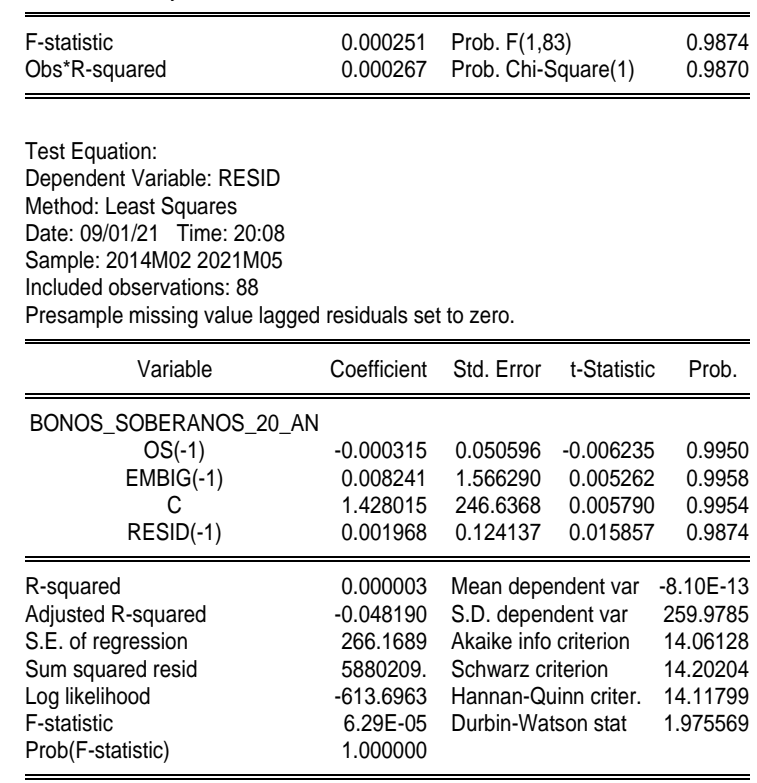

Tabla 3

Test de Heterocedasticidad de White Heteroskedasticity Test: White

\begin{tabular}{|c|c|c|c|c|}
\hline $\begin{array}{l}\text { F-statistic } \\
\text { Obs*R-squared } \\
\text { Scaled explained SS }\end{array}$ & $\begin{array}{l}0.785304 \\
4.021271 \\
4.165897\end{array}$ & \multicolumn{2}{|c|}{$\begin{array}{l}\text { Prob. F(5,82) } \\
\text { Prob. Chi-Square }(5) \\
\text { Prob. Chi-Square(5) }\end{array}$} & $\begin{array}{l}0.5632 \\
0.5464 \\
0.5258\end{array}$ \\
\hline \multicolumn{5}{|l|}{$\begin{array}{l}\text { Test Equation: } \\
\text { Dependent Variable: RESID^2 } \\
\text { Method: Least Squares } \\
\text { Date: 09/01/21 Time: } 20: 26 \\
\text { Sample: } 2014 \text { M02 2021M05 } \\
\text { Included observations: } 88\end{array}$} \\
\hline Variable & Coefficient & Std. Error & t-Statistic & Prob. \\
\hline 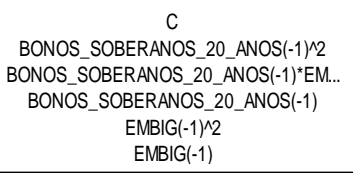 & $\begin{array}{r}477078.5 \\
0.027019 \\
-0.638629 \\
-227.3562 \\
3.212921 \\
3209.314\end{array}$ & $\begin{array}{l}754932.4 \\
0.029851 \\
0.659998 \\
288.1628 \\
9.728473 \\
3750.841\end{array}$ & $\begin{array}{r}0.631949 \\
0.905131 \\
-0.967622 \\
-0.788985 \\
0.330260 \\
0.855625\end{array}$ & $\begin{array}{l}0.5292 \\
0.3680 \\
0.3361 \\
0.4324 \\
0.7420 \\
0.3947\end{array}$ \\
\hline $\begin{array}{l}\text { R-squared } \\
\text { Adjusted R-squared } \\
\text { S.E. of regression } \\
\text { Sum squared resid } \\
\text { Log likelihood } \\
\text { F-statistic } \\
\text { Prob(F-statistic) }\end{array}$ & $\begin{array}{r}0.045696 \\
-0.012493 \\
119392.1 \\
1.17 \mathrm{E}+12 \\
-1150.494 \\
0.785304 \\
0.563202\end{array}$ & $\begin{array}{l}\text { Mean depend } \\
\text { S.D. depende } \\
\text { Akaike info cri } \\
\text { Schwarz crite } \\
\text { Hannan-Quin } \\
\text { Durbin-Watso }\end{array}$ & $\begin{array}{l}\text { lent var } \\
\text { int var } \\
\text { terion } \\
\text { rion } \\
\text { n criter. } \\
\text { in stat }\end{array}$ & $\begin{array}{l}79167.45 \\
118653.3 \\
26.28396 \\
26.45287 \\
26.35201 \\
2.362467\end{array}$ \\
\hline
\end{tabular}


Con la finalidad de comprobar que el modelo no presenta autocorrelación se realizó el test de Breusch-Godfrey serial de correlación LM. Al respecto, se puede concluir que el estadístico experimental del contraste tiene una probabilidad asociada de 0.9870 mayor de 0.05 y que el estadístico se ha situado en la región de aceptación, no se puede rechazar la hipótesis nula, es decir, no existe autocorrelación en el modelo estimado al 95\% de confianza, lo cual se puede verificar en la Tabla 2. De otro lado, con el propósito de verificar si existe heterocedasticidad se utilizó el test de White (Tabla 3), de lo cual podemos concluir que, dado que es mayor que el nivel de significancia del $5 \%$, no se puede rechazar la hipótesis nula de varianza constante en toda la muestra.

\section{DISCUSIÓN}

Después de analizar los resultados obtenidos respecto al grado de causalidad entre el riesgo país y el rendimiento de los bonos a 20 años emitidos por el gobierno peruano, se puede inferir que el activo financiero emitido por el MEF esta influenciado por el EMBIG, el cual mide el riesgo país. Tanto el rendimiento de los bonos rezagado en un periodo como variable endógena y el riesgo del Perú son significativas, por lo que ambos explican el comportamiento de la variable exógena de la presente investigación.

El importante destacar que la tenencia de los bonos soberanos ha estado principalmente en manos de los no residentes, hasta antes de la crisis política que viene experimentando el país, pasando del $51.4 \%$ a diciembre del 2020 al $49 \%$ de tenencia al primer trimestre del 2021, debido a la incertidumbre política y a la tendencia alcista de las tasas de interés producto de la política monetaria del Banco Central de Estados Unidos. De otro lado, independientemente del plazo de los bonos soberanos en América Latina, estos han tenido un crecimiento en sus rendimientos, sin embargo, las tasas de rendimiento del país son bajas en comparación a sus pares regionales.

Estos resultados concuerdan con investigaciones realizadas por el BCRP, donde el riesgo de los países emergentes influye en la tasa de rendimiento de un bono, así como las variables inflación e interés real. La inversión en un bono emitido por un mercado emergente presenta mayores riesgos de liquidez y de crédito, entre otros aspectos como: políticos y de tipo de cambio (Banco Central de Reserva del Perú, 2021). Así dentro de los factores que influyen el rendimiento de la bolsa de valores de Lima en el nivel de rentabilidad de los bonos soberanos a 30 años del Perú, periodo 2014-2018, las variables riesgo país, tasa de referencia y crecimiento de la economía, rendimiento de la bolsa de valores de Lima influyen en los activos financieros. (Quiñones, 2019).

\section{CONCLUSIONES}

Los bonos soberanos emitidos por el MEF a través del programa de creadores de mercado (PCM), para desarrollar un mercado de deuda pública en moneda nacional, ha fomentado el desarrollo del mercado de capitales local.

Dado el grado de inversión definido por las clasificadoras de riesgo del mundo, así como la evolución del riesgo país, han contribuido a una importante demanda de no residentes de los activos financieros emitidos por el sector público. 
En el análisis comparativo del rendimiento de los bonos soberanos a 20 años entre Perú y México, dado que no hay otro país en América Latina de igual plazo, se puede concluir que el Perú se encuentra por debajo de México, cuyo rendimiento hoy es superior en a $0.71 \%$, mientras que hace un año fue de $1.47 \%$, lo cual denota una reducción del $50 \%$.

Finalmente, tanto Chile como Perú tienen el riesgo país más bajo de Latinoamérica, el cual oscila entre 143.7 y 170 puntos básicos respectivamente.

\section{REFERENCIAS}

Banco Central de Reserva del Perú. (2021). Mercado de capitales. Reporte de estabilidad financiera, 39-54.

Gnecco, M. (2008). Riesgo país y tasa de corte en evaluación de proyectos. Revista FACES, 14(30), 25-45. http://nulan.mdp.edu.ar/1278/.

Gomero, N., Masuda, V., \& Barrera, J. (2014). Bonos soberanos y su relación con el escenario macroeconómico global. Quipukamayoc Revista de la Facultad de Ciencias Contables, 22(42), 31-43. https://revistasinvestigacion.unmsm.edu.pe/index.php/quipu/article/download/110 33/9923/.

Horna, I. (2020). Perspectivas del financiamiento corporativo y el mercado de valores del Perú. RETOS. Revista de Ciencias de la Administración y Economía, 10(19), 135-152. https://doi.org/10.17163/ret.n19.2020.08.

Levinson, M. (2008). Guía de Mercados Financieros. Ediciones Gestión 2000. https://books.google.com. pe/books?id=-1jqas$\mathrm{d} 77 \mathrm{oC} \&$ printsec $=$ frontcover $\&$ source=gbs_ge_summary_r\&cad=0\#v=onepage $\& q \& f=f$ alse.

Limas, S., \& Franco, J. (2018). El riesgo país para Colombia: interpretación e implicaciones para la economía y la inversión extranjera, 2012-2017. Revista Finanzas y Política Económica, 10(1), 153-171. https://doi.org/10.14718/revfinanzpolitecon.2018.10.1.6.

Ministerio de Economía y Finanzas. (23 de Noviembre de 2020). Obtenido de Ministerio de Economía y Finanzas: https://www.gob.pe/institucion/mef/noticias/315751-perucoloco-tres-nuevos-bonos-por-us-4-000-millones-incluyendo-una-emision-convencimiento-dentro-de-101-anos

Moreno, F. (2014). Bonos Financieros focalizados en los Bullet y en América. Revista Ciencia UNEMI, 7(11), 72-80.

http://ojs.unemi.edu.ec/index.php/cienciaunemi/article/view/114.

Pilbeam, K. (2018). Finance and Financial markets. Macmillan Publishers Limited. 
Quiñones, R. (2019). Factores macroeconómicos que afectan el rendimiento de los bonos soberanos d 30 años del Perú, periodo 2014-2018. Tacna, Perú.

Rodríguez, A., Ayala, J., \& Iturralde, T. (2003). La percepción del riesgo país en el caso Americano. ¿qué variables son relevantes? Revista Latinoamericana de Economía, 34(133), 101-128. https://www.redalyc.org/pdf/118/11825949012.pdf.

Rodríguez, V. (2010). Ofertas públicas primarias de bonos corporativos en el Perú. Quipukamayoc, 17(33), 71-95. https://doi.org/10.15381/quipu.v17i33.4554.

Rossini, R., Montoro, C., \& Luna, M. (Diciembre de 2020). Desarrollo del mercado financiero y política monetaria: la experiencia peruana. Estudios económicos, 9-20.

Santilli, E. (2021). El riesgo país en Sudamérica y México. Un estudio con datos de panel. Análisis económico, 36(91), 181-210.

http://www.scielo.org.mx/scielo.php?script=sci_arttext\&pid=S244866552021000100181.

Stowell, D. (2018). Investment Banks, Hedge Funds, and Private Equity. Editorial Academic Press. 